\title{
Comment étalonner et configurer efficacement les instruments intelligents et réussir à documenter ces données
}

\author{
Alex Maxfield1, Heikki Laurila2, Christophe Boubay3
}

1 Beamex Ltd, Newtown Grange Farm Business Park, Desford Road, Newtown Unthank, Leicestershire LE9 9FL, United Kingdom

2 Beamex Oy Ab, Ristisuonraitti 10, 68600 Pitarsaari, Finland

3 Beamex Sas, 253 Boulevard de Leeds, 59777 Lille, France

\begin{abstract}
A process transmitter is a device that senses a physical parameter (pressure, temperature, etc.) and generates an output signal proportional to the measured input. The term "smart" is more of a marketing term than a technical definition. There is no standardised technical definition for what smart really means in practice. Generally, in order for a transmitter to be called smart, it will utilize a microprocessor and should also have a digital communication protocol that can be used for reading the transmitter's measurement values and for configuring various settings in the transmitter. A microprocessorbased smart transmitter has a memory that can perform calculations, produce diagnostics, etc. Furthermore, a modern smart transmitter typically outperforms an older type of conventional transmitter regarding measurement accuracy and stability. For the engineers who need to configure and calibrate the transmitter, the digital communication protocol and the open access to a myriad of critical configuration parameters are the biggest differences compared to conventional analogue only transmitters and as a result of these differences there is a significant risk to process safety, quality and metrology if good practice is ignored.
\end{abstract}

Les instruments fonctionnant avec des protocoles numériques dits " intelligents ", sont de plus en plus répandus dans l'industrie de transformation. La majorité des instruments actuellement proposés sur le marché sont des instruments intelligents. Ces nouveaux instruments intelligents imposent de nouvelles exigences aux processus d'étalonnage et de configuration et apportent également de nouveaux défis, surtout lorsqu'ils sont mis en lieu et place d'une installation existante, qui, elle est purement analogique.

Figure $1:$ Chronologie des technologies de

l'instrumentation / Source : www.fieldbus.org

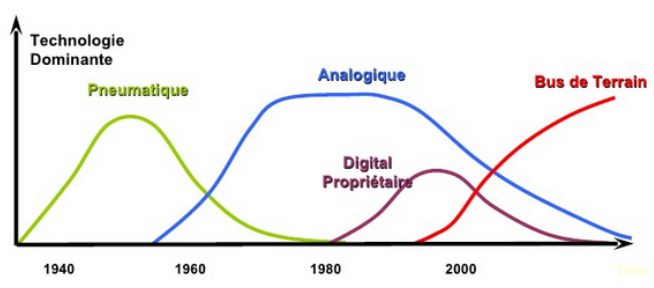

\section{Qu'est-ce qu'un transmetteur "intelligent"?}

Un transmetteur de process est un appareil qui détecte un paramètre physique (pression, température, débit, conductivité, niveau, etc.) et produit un signal de sortie proportionnel à l'entrée mesurée. Le terme "intelligent" est davantage un terme mercantile qu'une définition technique. Il n'existe aucune définition technique standardisée de la signification réelle du terme " intelligent " dans la pratique.

\section{Mais pourquoi utilise-t-on des instruments intelligents ?}

Généralement, pour qu'un transmetteur soit qualifié d'intelligent, il doit utiliser un microprocesseur et doit également posséder un protocole de communication numérique qui peut être utilisé pour lire les valeurs 
mesurées par le transmetteur et pour configurer différents paramètres de celui-ci. De plus, un transmetteur intelligent moderne surpasse généralement les transmetteurs classiques de type plus ancien sur le plan de la précision et de la stabilité des mesures. Ainsi, un transmetteur analogique, généralement avec un courant de sortie de $4 / 20 \mathrm{~mA}$, aura tendance à dériver davantage dans le temps qu'un instrument intelligent. De plus, ces protocoles numériques permettent aux fabricants de standardiser leur production puisque les échelles deviennent entièrement configurables, ce qui apporte également un gain pour les utilisateurs en terme de gestion des stocks.

Le fait que les instruments soient équipés d'un microprocesseur offre l'avantage de pouvoir transmettre de nombreuses données et non pas uniquement une retranscription de la mesure de la grandeur physique d'entrée. Ainsi, les instruments intelligents ont la possibilité de fournir des informations de diagnostic ou encore de réaliser des calculs.

L'utilisation de protocole numérique permet également le transfert de données sur un seul segment de câble et assure également une meilleure intégrité de ces dernières puisque le transfert est binaire, soit les données sont reçues soit elles se perdent.

Dans tous les cas, pour les techniciens qui doivent configurer et étalonner les transmetteurs, le protocole de communication numérique constitue la plus grosse différence par rapport aux transmetteurs analogiques. Les techniciens ne peuvent plus simplement mesurer le signal de sortie analogique mais ils doivent avoir la possibilité de communiquer avec le transmetteur et de lire le signal numérique. Cela amène un problème à résoudre entièrement nouveau : comment lire la sortie numérique?

\section{Quels sont les protocoles existants et quels nouveaux défis apportent-ils ?}

Il existe différents protocoles numériques parmi les transmetteurs considérés comme intelligents. Certains sont des protocoles propriétaires développés par les fabricants mais ces derniers semblent perdre du terrain au profit de protocoles reposant sur des normes ouvertes en raison de l'interopérabilité qu'ils permettent. La plupart des protocoles reposent sur des normes ouvertes. Le protocole de transmetteur le plus courant actuellement est le protocole HART (Highway Addressable Remote Transducer). Un transmetteur HART contient à la fois un signal $\mathrm{mA}$ analogique classique et un signal numérique superposé au signal analogique. Etant donné qu'il contient également le signal analogique, il est compatible avec les installations classiques. Le protocole HART semble gagner de l'ampleur depuis quelque temps grâce au tout dernier protocole HART sans fil, le WirelessHart.

Figure 2 : Diagramme de câblage des transmetteurs HART et WirelessHart / Source : Beamex

Calibration World Magazine Winter 2014 issue

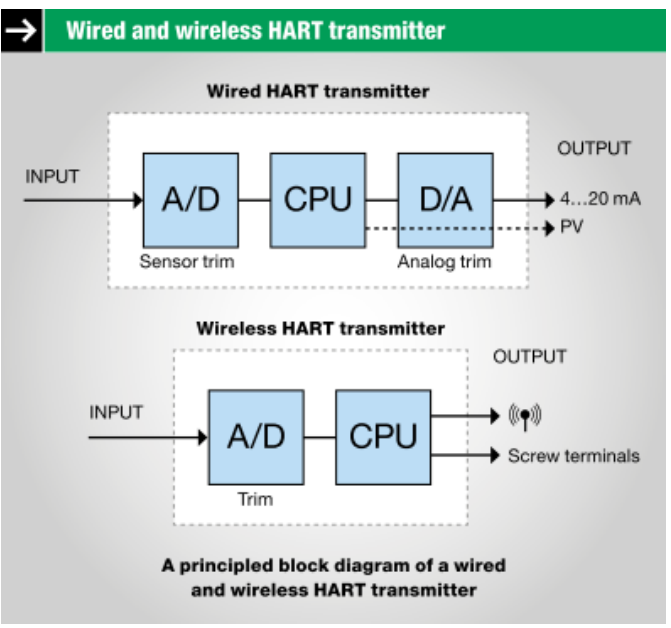

Les bus de terrain quant à eux, ne contiennent qu'une sortie numérique mais pas de signal analogique. Ainsi, on trouve les protocoles FOUNDATION Fieldbus et Profibus PA. L'architecture de ces protocoles est composée de différents blocs ayant chacun une fonction bien particulière :

- Le Resource Block définit les paramètres relatifs à l'application du capteur, tels que l'identifiant fabricant, le type d'instrument, ...

- Le Function Block regroupe les fonctions de contrôle du transmetteur tel que le PID

- Le Transducer Block offre une interface pour les capteurs de température, pression, débit ou encore les vannes.

Figure 3 : Blocs composants le protocole Foundation Fieldbus Source : www.fieldbus.org 


\section{Blocks}
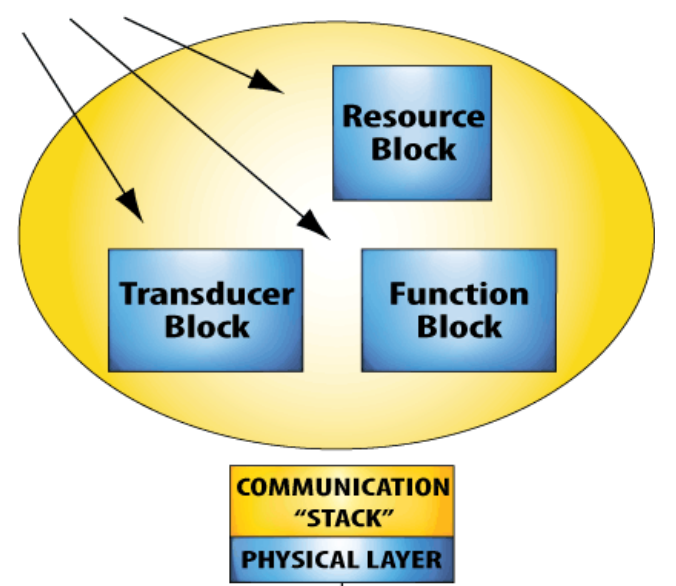

Fieldbus

Contrairement aux idées reçues, un instrument intelligent, tout comme un instrument purement analogique, à tendance à dériver avec le temps. L'apport de protocole numérique ne diminue donc pas la périodicité d'étalonnage de ces capteurs et n'exclue, en aucun cas, pas le besoin d'étalonner les capteurs.

\section{Quelle sont les méthodes de configuration et d'étalonnage ?}

Avant toutes choses, il est important de rappeler rapidement un problème de terminologie qui provoque souvent certaines confusions : la signification des termes et la différence entre configuration et étalonnage.

Selon les normes internationales, l'étalonnage est une comparaison du dispositif testé avec un instrument de référence traçable (un étalon de référence/calibrateur) et la production de la documentation de cette comparaison. Bien que formellement l'étalonnage ne comporte aucun réglage, en pratique, le réglage est souvent inclus dans le processus d'étalonnage. Donc, pour effectuer l'étalonnage d'un instrument intelligent, il faut un dispositif de référence métrologique traçable (un calibrateur).

La configuration signifie l'utilisation du protocole de communication numérique pour modifier des paramètres à l'intérieur de l'instrument. Ces paramètres peuvent être l'unité physique, la gamme d'utilisation, l'amortissement, le repère pour le nommer dans la boucle ou sur un système de supervision, etc. La configuration peut se faire à l'aide d'un logiciel de configuration ou d'un communicateur portable. Il est important de se souvenir qu'un communicateur seul ne peut pas être utilisé pour l'étalonnage métrologique afin de vérifier l'exactitude d'un instrument. La configuration de paramètres d'un transmetteur intelligents avec un communicateur n'est pas un étalonnage et ne garantit pas la justesse d'un point de vu technique mais surtout légal. Pour un étalonnage métrologique réel, un standard de référence traçable (un calibrateur) est toujours nécessaire. La configuration n'est pas la même chose que l'étalonnage.

Avec l'utilisation de ces protocoles de communication numériques, de nouvelles révisions apportant ou modifiant les fonctionnalités des instruments intelligents sont intégrées. Il devient donc nécessaire d'avoir un système de communication et de configuration qui soit lui-même à jour. Ainsi le choix du configurateur est important dans la pérennité de la solution car il faut être certain de pouvoir installer les mises à jour des instruments mais également d'avoir un communicateur qui possède les derniers pilotes pour suivre les évolutions constantes apportées par les fabricants. Aujourd'hui, les moyens de configuration existants sont :

- Un ordinateur sur lequel un logiciel de communication et configuration est installé.

- Une pocket de communication permettant de configurer l'instrument sur le terrain.

- Un calibrateur et communicateur qui, aura les mêmes fonctions qu'une pocket mais qui pourra également réaliser l'étalonnage de ces derniers.

En ce qui concerne les instruments classiques, selon les normes internationales, l'étalonnage consiste à comparer l'appareil qui fait l'objet des tests et un instrument de référence traçable (calibrateur) et à documenter la comparaison. Si l'étalonnage est réalisé avec un calibrateur de documentation, il documente automatiquement les résultats de l'étalonnage. Pour étalonner un transmetteur analogique classique, il est possible de produire ou de mesurer l'entrée du transmetteur en mesurant simultanément sa sortie. Dans ce cas, l'étalonnage est assez facile ; il faut un calibrateur à fonction double capable de traiter simultanément l'entrée et la sortie process ou alors deux calibrateurs à fonctions simples distinctes.

Cependant, comment est-il possible d'étalonner un transmetteur intelligent avec la sortie qui est un signal numérique ? Bien évidemment, l'entrée du transmetteur doit continuer à être générée/mesurée de la même manière qu'avec un transmetteur classique, c'est-à-dire avec un calibrateur. Toutefois, pour voir ce qu'est la sortie du transmetteur, il faut un appareil ou un logiciel capable de lire et d'interpréter le protocole numérique. L'étalonnage peut par conséquent être une tâche très difficile; plusieurs types 
d'appareils peuvent être nécessaires et il faut plusieurs personnes pour exécuter le travail. Il est parfois très difficile, voire impossible, de trouver un appareil qui convienne, en particulier s'il s'agit d'un appareil portable permettant de réaliser des étalonnages sur le terrain.

Le protocole HART câblé (par opposition au protocole WirelessHart) est un protocole hybride qui comprend une communication numérique superposée à un signal de sortie analogique 4-20 mA classique. Le signal de sortie 4-20mA d'un transmetteur HART câblé est étalonné de la même manière qu'un transmetteur classique. Toutefois, pour ajuster un tel instrument et au regard du schéma fonctionnel de la figure 2, il est nécessaire d'agir sur les deux blocs de conversions :

- l'analogique/numérique, qui convertit le signal d'entrée en signal HART, communément appelé "sensor trim",

- le numérique/analogique, qui convertit le signal HART en $4 / 20 \mathrm{~mA}$, nommé "'analog trim',

Ainsi, deux opérations d'ajustage sont nécessaires. En exemple, on peut citer l'ajustage d'un transmetteur de pression pour lequel on pourrait utiliser les matériels suivants :

- Une pompe avec un manomère, afin de générer le signal d'entrée et d'obtenir une référence.

- Une alimentation 24V pour la boucle.

- Une pocket HART avec une résistance $250 \mathrm{ohm}$ afin de lire le signal numérique. A ce stade, il est possible de réaliser le sensor trim.

- Ajouter à cela un multimètre pour mesurer la sortie $4 / 20 \mathrm{~mA}$ et ainsi ajuster le signal analogique, analog trim.

$\mathrm{Ou}$ alors utiliser un calibrateur et communicateur multifonctions qui serait capable de réunir toutes ces fonctions en un seul appareil et même potentiellement documenter l'intervention si ce dernier est un calibrateur de documentation. l'exactitude des informations puisqu'ils sont plus facilement traçable et remontent plus de données.

Bien que les données des instruments purement analogiques soient généralement reliées à une supervision et soient enregistrées, les instruments intelligents offrent la possibilité de réaliser des diagnostics ou encore de créer des alarmes internes. Ainsi, il est possible, par exemple, d'enregistrer la date du dernier étalonnage dans l'instrument ou encore de retrouver les différentes configurations qui lui ont été attribuées (échelle, unité, ...) grâce aux mêmes solutions que celles permettant la configuration. Tout cela simplifie grandement le remplacement d'un instrument obsolète et la planification des interventions.

Il est essentiel de maîtriser son process de fabrication afin de répondre aux normes en vigueur et aux nombreux audits auxquels doivent faire face les industries, ce qui rend donc primordial l'assurance de la documentation des interventions réalisées sur les instruments, aussi bien en terme d'action préventive, tel qu'un étalonnage, que curative, tel qu'un remplacement de capteur. Et grâce aux instruments intelligents, la documentation et, par conséquent, la traçabilité tendent à être simplifiées et à assurer la transparence. Comme les configurations des capteurs sont documentées, il est également plus aisé de remplacer un instrument défaillant puisque les protocoles numériques permettent de transférer directement la configuration de l'ancien instrument dans celui venant en lieu et place, tout en gardant les mêmes repères. A cela, la traçabilité peut être automatiquement réalisée par des systèmes informatiques adéquats puisque le nouveau capteur aura certes la même configuration mais il possédera un numéro d'identification propre et unique. Bien entendu, il faudra tout de même étalonner ce nouvel instrument et documenter cette vérification avec un calibrateur lui-même traçable dans la chaîne métrologique.

\section{Comment peut-on assurer la traçabilité et documenter efficacement les interventions réalisées sur ces instruments?}

La documentation et la traçabilité des données sont de plus en plus présentes dans l'industrie de transformation car elles assurent le suivi, la maîtrise et la qualité du process, et en ce sens, les instruments intelligents aident à garantir 